\title{
Prevalência de dor nas costas e hábitos posturais inadequados em escolares do Ensino Fundamental do Colégio de Aplicação da UFRGS
}

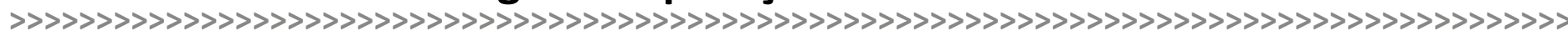

\author{
Tássia Silveira Furlanetto* \\ Fernanda da Silva Medeiros** \\ Cláudia Tarragô Candotti***
}

\begin{abstract}
Resumo:
O objetivo do estudo foi verificar a prevalência e a associação da dor nas costas e dos hábitos posturais inadequados em escolares do Ensino Fundamental do Colégio de Aplicação da Universidade Federal do Rio Grande do Sul. 197 escolares (a partir do 5o Ano) foram avaliados com o questionário Back Pain and Body Posture Evaluation Instrument (BackPEI), o qual visa identificar a presença de dor nas costas e avalia fatores de risco demográficos (idade e sexo) e comportamentais (hábitos de vida diários). Os resultados demonstraram uma prevalência de dor nas costas dos escolares de 55,3\%, tendo associação significativa com o sexo feminino. Foi encontrada prevalência superior a $90 \%$ de hábitos posturais inadequados nas atividades de sentar para escrever, sentar em uma cadeira e/ou banco, sentar para utilizar o computador e pegar objeto do chão. Houve associação significativa entre sentar para escrever inadequadamente e dormir adequadamente com a dor nas costas.
\end{abstract}

\section{Palavras-chave:}

Dor nas costas. Hábitos posturais. Saúde da criança e do adolescente.

\begin{abstract}
:
The aim of the study was to assess the prevalence and the association between back pain and inadequate postural habits in elementary school students of the Colégio de Aplicação, Federal University of the Rio Grande do Sul. 197 students were evaluated with the BackPEI questionnaire (Back Pain and Body Posture Evaluation Instrument), which aims to identify the presence of back pain and assesses demographic risk factors (age and gender) and behavioral factors (habits of daily life). The results showed a prevalence of back pain in students at the $55.3 \%$, with a significant association with the female gender. Higher prevalence (more than $90 \%$ ) of inadequate postural habits was found in sitting position to write, sitting position in a chair and/or bench, sitting position to use the computer and pick up object from floor. There was a significant association between inadequate sitting position to write and adequate sleep position with back pain.
\end{abstract}

\section{Keywords:}

Back pain. Postural habits. Children and adolescents health.

\footnotetext{
* Doutoranda do Programa de Pós Graduação Ciência do Movimento Humano da Universidade Federal do Rio Grande do Sul. E-mail: tassiasf@gmail.com

** > Graduada em Fisioterapia da Universidade Federal do Rio Grande do Sul. E-mail: fernanda.fsmedeiros@gmail.com *** > Doutora e professora do Departamento de Educação Física da Escola de Educação Física, Fisioterapia e Dança da Universidade Federal do Rio Grande do Sul.

E-mail: claudia.candotti@ufrgs.br
} 


\section{Introdução}

A dor nas costas é um distúrbio comumente encontrado nas sociedades industrializadas, afetando de $54 \%$ a $90 \%$ da população adulta (NAJENSON et al., 2010; FERREIRA et al., 2011) e de 54\% a $74 \%$ (NOLL et al., 2012; SHEHAB; JARALLAH, 2005) da população de crianças e de adolescentes. Além disso, é estimado que cerca de 70 a $85 \%$ da população terá, no decorrer da vida, algum episódio álgico na coluna vertebral (HOY et al., 2010; FERREIRA et al., 2011).

Atualmente, a dor nas costas em escolares é motivo de preocupação, não somente pela alta prevalência (MARTINEZ-CRESPO et al., 2009; SKOFFER, 2007), mas especialmente porque essa dor parece estar interligada ao estilo de vida dos jovens, o qual inclui, dentre outras questões, os hábitos posturais adotados durante a vida e as alterações na postura corporal estática (BRACCIALLI; VILARTA, 2000).

Nesse sentido, a causa da dor nas costas na população de crianças e de adolescentes tem sido demonstrada como multifatorial, abrangendo fatores físicos, comportamentais, genéticos e psicossociais (KOROVESIS et al., 2005). Dentre os principais fatores associados às dores nas costas em escolares, podem ser apontados: o sexo, o nível socioeconômico e os hábitos posturais inadequados durante a realização de atividades cotidianas, mantidas por tempo prolongado (BUENO; RECH, 2013; LIS et al., 2007; MARTÍNEZ-CRESPO et al., 2009; AUVINEN et al., 2010).

Embora a dor nas costas de escolares seja um acometimento recorrente nessa população, a nível mundial, e considerado como um problema de saúde pública, não se pode generalizar sua prevalência em uma determinada região para demais regiões, mesmo dentro de um único país. Isso se deve a sua natureza multifatorial. Assim, estudos que investiguem a prevalência da dor nas costas em diferentes regiões do país são importantes ferramentas para a elaboração de políticas públicas de saúde à criança e ao adolescente. Ainda, se considerar a lombalgia na infância e na juventude como preditora da lombalgia na vida adulta (WATSON et al., 2002), faz-se ainda mais necessário o conhecimento dos fatores que contribuem para a ocorrência de dor nas costas de forma precoce, visando ao desenvolvimento de estratégias preventivas mais eficazes (HOY et al., 2010). Nessa perspectiva, justifica-se a realização do presente estudo, que tem como objetivo verificar a prevalência e a associação da dor nas costas e de hábitos posturais inadequados em escolares do Ensino Fundamental do Colégio de Aplicação da Universidade Federal do Rio Grande do Sul, Porto Alegre/RS.

\section{Metodologia}

O tamanho da amostra foi calculado com base nos dados reportados por Silva (2013), os quais apontam a prevalência de dor nas costas em 72,4\% dos escolares. Para esse cálculo, foi utilizado um nível de confiança de $95 \%$ e um erro amostral de $10 \%$. Prevendo, ainda, uma perda amostral e desistência de $30 \%$, calculou-se que seria necessário um número mínimo de 135 escolares para cumprir com os propósitos do presente estudo.

Foram convidados a participar do estudo todos os escolares do Ensino Fundamental, a partir do 5o Ano, do Colégio de Aplicação da Universidade Federal do Rio Grande do Sul (UFRGS). Todos os pais e/ou responsáveis dos escolares consentiram que os estudantes participassem do estudo, por intermédio da assinatura do Termo de Consentimento Livre e Esclarecido. O projeto foi aprovado pelo Comitê de Ética e Pesquisa da UFRGS e pela COMPESQ do Colégio de Aplicação, respeitando a Resolução 466/2012 do Conselho Nacional de Saúde. 
Os escolares foram selecionados de acordo com os seguintes critérios: a) estarem matriculados e cursando a partir do 5o Ano do Ensino Fundamental do Colégio de Aplicação da UFRGS no ano de 2013; b) possuírem de 10 a 18 anos de idade no ano de 2013 e c) aceitarem participar do estudo, voluntariamente. Foram excluídos da amostra os escolares que optaram em desistir de participar do estudo ao longo do momento avaliativo, ou ao longo das análises de dados e escolares, cujos responsáveis não autorizaram sua participação.

A coleta de dados ocorreu durante as aulas de Educação Física, sendo agendada previamente com os professores da disciplina. Por ocasião desse agendamento, os escolares receberam o Termo de Consentimento Livre e Esclarecido, o qual deveria ser levado aos seus responsáveis e trazido no próximo dia de aula, quando ocorreria a coleta de dados. Um único avaliador foi responsável pela aplicação dos questionários em cada turma individualmente, no próprio local de aula. Inicialmente, após receber de cada escolar o termo devidamente assinado, o avaliador explicou coletivamente como deveria ser preenchido o questionário, o qual, a seguir, foi distribuído para cada aluno e respondido individualmente. $\mathrm{O}$ pesquisador permaneceu no local durante o preenchimento dos questionários, que durou, em média, 20 minutos, recolhendo-os no momento em que todos os escolares terminassem.

Para identificar a prevalência de dor nas costas e de hábitos posturais, foi utilizado um questionário auto-aplicável, válido e reprodutível, denominado Back Pain and Body Posture Evaluation Instrument (BackPEI) (NOLL et al., 2013). O BackPEI é um questionário constituído por 21 questões fechadas e possui uma versão para cada sexo, podendo ser acessado pelo site <http://www.ufrgs.br/biomec/materiais.html >. O instrumento visa identificar a presença de dor nas costas nos últimos três meses e avalia fatores de risco demográficos (idade e sexo) e comportamentais (nível de exercício físico e prática competitiva de exercício físico; horas diárias assistindo à televisão e utilizando o computador; horas diárias de sono e hábito de ler e/ou estudar na cama; postura para dormir, sentar na cadeira para escrever, sentar na cadeira para conversar, utilizar o computador e ao pegar objeto do solo; meio de transporte utilizado para transportar o material escolar e modo de transporte da mochila escolar) (NOLL et al., 2013). Especificamente, as questões 1 a 3 referem-se à prática regular de exercícios físicos, a frequência dessa prática e se essa prática ocorre de maneira competitiva ou não. As questões 4 a 8 se referem ao tempo transcorrido, praticando hábitos de vida sedentários. As questões que envolvem os hábitos posturais (questões 9 a 14) são todas ilustrativas com fotografias, em que o escolar deve assinalar aquela que mais representa sua postura cotidiana. As questões de 15 a 17 mencionam possíveis fatores hereditários que podem vir a influenciar a presença de dores nas costas dos escolares. E as últimas questões (18 a 21) avaliam a presença, intensidade e frequência da dor nas costas nos escolares.

As respostas do questionário foram codificadas e tabuladas para posterior análise estatística. Em relação à realização dos hábitos posturais, em cada questão, apenas uma alternativa foi considerada adequada, justamente aquela em que a posição dos segmentos corporais ilustrados na imagem apresentava uma postura adequada. Todas as demais alternativas foram consideradas inadequadas.

Para a análise estatística foi utilizado o Software SPSS (versão 18.0). Os dados foram analisados a partir de estatística descritiva, sendo os resultados apresentados por meio de frequências. Foi realizado também o cálculo das Razões de Prevalência (RP) e de seus respectivos Intervalos de Confiança de 95\% (IC95\%). As RP foram calculadas por meio de uma análise multivariada realizada a partir do modelo de Regressão de Poisson, com variância robusta $(\alpha=0,05)$. A variável dependente foi a presença de dor nas costas e as variáveis independentes foram os hábitos posturais adotados pelos indivíduos e o sexo. 


\section{Resultados}

Participaram do estudo 197 escolares, cujas médias de idade, estatura e massa corporal foram: $13,4( \pm 1,3)$ anos, $1,6( \pm 0,1) \mathrm{m}$ e 54,3 $( \pm 13) \mathrm{kg}$, respectivamente. A amostra de escolares ( $n=197)$ foi composta por 107 meninos (54,3\%) e 90 meninas (45,7\%). A frequência e o percentual dos escolares estratificados por sexo e idade estão apresentados na Tabela 1.

Tabela 1 - Frequência e percentual dos escolares avaliados estratificados por sexo e idade.

\begin{tabular}{|l|l|l|l|}
\hline \multicolumn{1}{|c|}{ Idade (anos) } & \multicolumn{1}{|c|}{$\begin{array}{c}\text { Masculino } \\
\mathbf{n ~ ( \% )}\end{array}$} & \multicolumn{1}{|c|}{$\begin{array}{c}\text { Feminino } \\
\mathbf{n ~ ( \% )}\end{array}$} & \multicolumn{1}{|c|}{$\begin{array}{c}\text { Total } \\
\mathbf{n}(\%)\end{array}$} \\
\hline 11 & $9(8,4)$ & $2(2,20)$ & $11(5,6)$ \\
\hline 12 & $23(21,5)$ & $27(30)$ & $50(25,4)$ \\
\hline 13 & $26(24,3)$ & $15(16,7)$ & $41(20,8)$ \\
\hline 14 & $29(27,1)$ & $28(31,1)$ & $57(28,9)$ \\
\hline 15 & $16(15)$ & $15(16,7)$ & $31(15,7)$ \\
\hline $15-18$ & $4(3,7)$ & $3(3,3)$ & $7(3,6)$ \\
\hline Total & $107(100)$ & $90(100)$ & $197(100)$ \\
\hline
\end{tabular}

Fonte: Dados da pesquisa, 2013.

A prevalência de dor nas costas dos escolares nos últimos três meses foi de 55,3\% $(n=109)$. Quando os escolares foram separados por sexo, 43\% $(n=46)$ dos meninos e $70 \%$ das meninas $(n=63)$ apresentaram dor nas costas, demonstrando que as meninas apresentam um percentual mais elevado de dor nas costas. Houve uma associação significativa $(\mathrm{p}=0,004)$ entre sexo e dor nas costas, sendo que as meninas têm mais chances de apresentar dor (Tabela 2). A Figura 1 apresenta a prevalência de dor nas costas nos últimos três meses, estratificada por sexo e idades.

Figura 1 - Resultados da prevalência de dor nas costas nos últimos três meses e distribuição dos escolares por sexo e idade.

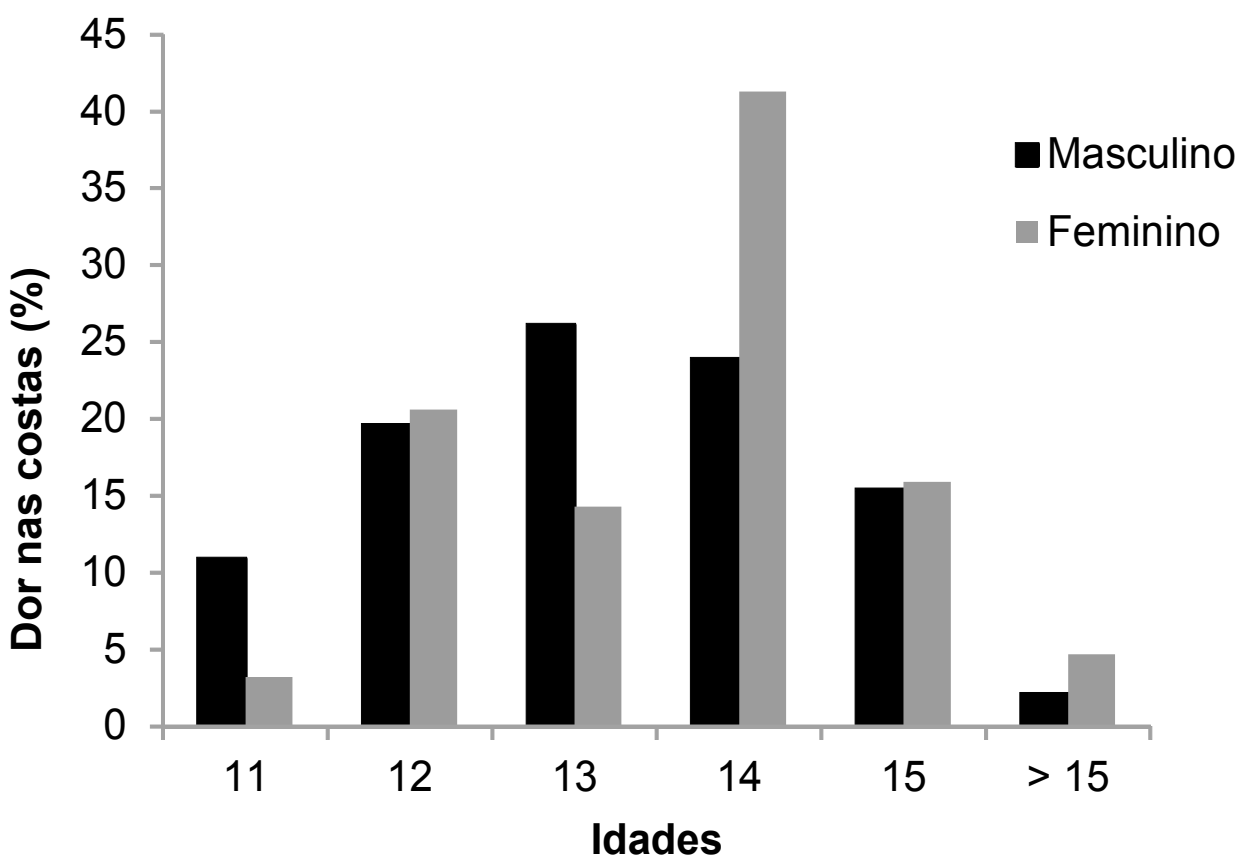

Fonte: Dados da pesquisa, 2013.

Do total de escolares avaliados, $80,2 \%(n=158)$ realizavam algum exercício físico ou esporte regularmente, seja ele na escola ou fora dela. Em contrapartida, apenas 19,8\% $(n=39)$ não praticavam exercício físico regularmente. 
Em relação aos hábitos posturais, os resultados, em geral, demonstraram alta prevalência de hábitos inadequados (acima de $90 \%$ dos escolares) nas seguintes atividades de vida diária (AVD’s): sentar para escrever, sentar em uma cadeira e/ou banco, sentar para utilizar o computador e pegar objeto do chão (Tabela 2). Em contrapartida, os resultados demonstraram prevalência superior de hábitos posturais adequados em relação aos inadequados nas AVD's dormir e modo de transportar o material escolar (Tabela 2). Considerando que algumas questões do questionário não foram respondidas pelo total de escolares participantes ( $n=197)$, a análise apresenta diferentes " $n$ " válidos para cada questão (Tabela 2).

Quanto aos resultados referentes à associação entre dor nas costas e hábitos posturais inadequados, na Tabela 2 pode-se perceber que houve uma associação significativa entre: (1) sentar para escrever inadequadamente e a dor nas costas e (2) dormir adequadamente e a dor nas costas.

Tabela 2 - Resultados de associação $\left(x^{2}\right)$ e razões de prevalência (RP) para a variável dependente (dor nas costas) e para as variáveis independentes (sexo e hábitos posturais).

\begin{tabular}{|c|c|c|c|c|}
\hline Variáveis (n) & $\begin{array}{l}\text { Total } \\
\text { n (\%) }\end{array}$ & $\begin{array}{l}\text { Presença de dor } \\
\text { nas costas n (\%) }\end{array}$ & $x^{2 a}$ & RP (IC 95\%) \\
\hline \multicolumn{5}{|l|}{ Sexo $(n=197)$} \\
\hline Masculino & $107(54,3)$ & $46(43)$ & $0,004^{*}$ & 1 \\
\hline Feminino & $90(45,7)$ & $63(70)$ & & $1,177(1,055-1,313)$ \\
\hline \multicolumn{5}{|l|}{$\begin{array}{l}\text { Prática do exercício físico } \\
\text { e/ou esporte }(\mathrm{n}=197)\end{array}$} \\
\hline Sim & $158(80,2)$ & $21(19,3)$ & 0,568 & $0,966(0,858-1,088)$ \\
\hline Não & $39(19,8)$ & $88(80,7)$ & & 1 \\
\hline \multicolumn{5}{|l|}{$\begin{array}{l}\text { Postura sentada } \\
\text { para escrever }(n=193)\end{array}$} \\
\hline Adequada & $10(5,2)$ & $3(2,8)$ & $0,049^{*}$ & 1 \\
\hline Inadequada & $183(94,8)$ & $105(97,2)$ & & $1,188(1,000-1,410)$ \\
\hline \multicolumn{5}{|l|}{$\begin{array}{l}\text { Postura sentada para } \\
\text { conversar com amigos }(n=194)\end{array}$} \\
\hline Adequada & $9(4,6)$ & $3(2,8)$ & 0,706 & 1 \\
\hline Inadequada & $185(95,4)$ & $105(97,2)$ & & $1,039(0,853-1,264)$ \\
\hline \multicolumn{5}{|l|}{$\begin{array}{l}\text { Postura sentada para utilizar } \\
\text { o computador }(\mathrm{n}=194)\end{array}$} \\
\hline Adequada & $18(9,3)$ & $6(5,6)$ & 0,325 & 1 \\
\hline Inadequada & $176(90,7)$ & $101(94,4)$ & & $1,079(0,928-1,254)$ \\
\hline \multicolumn{5}{|l|}{$\begin{array}{l}\text { Postura para pegar objeto } \\
\text { do solo }(n=196)\end{array}$} \\
\hline Adequada & $11(5,6)$ & $7(6,5)$ & 0,199 & $0,852(0,667-1,088)$ \\
\hline Inadequada & $185(94,4)$ & $101(93,5)$ & & 1 \\
\hline \multicolumn{5}{|l|}{$\begin{array}{l}\text { Meio e modo de transportar } \\
\text { o material escolar }(n=189)\end{array}$} \\
\hline Adequada & $100(52,9)$ & $51(49,5)$ & 0,747 & $0,983(0,883-1,093)$ \\
\hline Inadequada & $89(47,1)$ & $52(50,5)$ & & 1 \\
\hline \multicolumn{5}{|l|}{ Postura para dormir $(n=192)$} \\
\hline Adequada & $133(69,3)$ & $67(63,2)$ & $0,047^{*}$ & $1,113(1,001-1,237)$ \\
\hline Inadequada & $59(30,7)$ & $39(36,8)$ & & 1 \\
\hline
\end{tabular}




\section{Discussão}

A prevalência de dor nas costas nos escolares do Colégio de Aplicação da UFRGS $(55,3 \%)$ encontra-se dentro das taxas mundiais de prevalência de dor em crianças e em adolescentes, que variam de 54\% a 74\% (NOLL et al., 2012; SHEHAB; JARALLAH, 2005), e das taxas de prevalência de dor em escolares que variam de 20\% a 70\% (SKOFFER, 2007; VITTA et al., 2011; FERREIRA et al., 2011; ZAPATA et al., 2006; NOLL et al., 2012; NOLL et al., 2013a; SILVA, 2013).

Achados similares, demonstrando maior prevalência de dor nas costas no sexo feminino, foram encontrados em estudos anteriores (VITTA et al., 2011; SHEHAB; JARALLAH, 2005; WATSON et al., 2002; NOLL et al., 2012). Essa diferença entre os sexos pode ser explicada por algumas suposições. A primeira relaciona-se com as características anatomofuncionais distintas em relação aos meninos, como menor força física, fazendo com que o gasto energético das meninas seja maior quando expostas à demanda de trabalho similar, aumentando o risco de sobrecarga musculoesquelética (NOLL et al., 2012; VITTA et al., 2011). A segunda é de ordem psicossocial e se dá pelo fato das mulheres apresentarem, geralmente, um limiar de dor inferior e se queixarem com maior frequência do que os homens (NOLL et al., 2012; VITTA et al., 2011). Além disso, tem sido referido que as mulheres possuem maior permissão social para expor seus sintomas e sentimentos, devendo-se, ambos os eventos, a fatores sociais e educacionais (VITTA et al., 2011).

Tem sido relatado que os hábitos posturais inadequados, durante a prática das AVD's, podem ser uma das possíveis causas da alta prevalência de dor em escolares (BRACCIALLI; VILARTA, 2000). Dentre os hábitos posturais avaliados no presente estudo, as atividades de sentar para escrever, sentar em uma cadeira e/ou banco, sentar para utilizar o computador e pegar objeto do chão apresentaram alta prevalência de postura inadequada para sua realização. Corroborando com o presente estudo, Noll et al. (2013a) encontrou prevalência superior a $80 \%$ de postura inadequada nas posições sentadas para escrever, em uma cadeira/banco e ao utilizar o computador e prevalência superior a $90 \%$ ao pegar objeto do chão. Detsch et al. (2007) também demonstrou que apenas 27,5\% dos avaliados apresentaram hábitos posturais adequados ao utilizar o computador.

Ainda, nesse sentido, pode-se observar uma associação entre dor nas costas e a postura inadequada de sentar para escrever. Com base nos dados em relação à postura sentada, os achados tornam-se preocupantes, uma vez que os escolares, em geral, permanecem, em média, de 4 a 5 h diárias na posição sentada durante os períodos regulares de ensino, 5 vezes por semana (FREIRE; TEIXEIRA; SALES, 2008). Além disso, em muitas outras AVD's, como assistir à televisão e utilizar o computador, os escolares permanecem em uma posição sentada, podendo adotar esta postura inadequada durante $8 \mathrm{~h}$ diárias, sendo um importante fator de risco para a ocorrência de alterações posturais (MARTÍNEZ-CRESPO et al., 2009). Um exemplo disso foram os achados de Kunzler (2013) que, ao analisar se existe uma associação entre alteração postural e postura inadequada para escrever, verificou a existência de uma associação significativa entre alteração postural da curvatura torácica e a postura de sentar para escrever.

Ainda, a postura sentada está associada a uma maior pressão nos discos vertebrais que a posição em pé; e a manutenção prolongada da postura inadequada de sentar pode trazer mais alguns malefícios, tais como: pouco movimento dos membros tornando a atividade física insuficiente, problema de circulação sanguínea nos membros inferiores, predisposição a maiores índices de fadiga e formigamento em diferentes partes do corpo e, principalmente, a processos degenerativos nas estruturas osteoarticulares da coluna vertebral (CARVALHO et al., 2008; CANDOTTI; NOLL; CRUZ, 2010). 
Não obstante, outra AVD que, apresentou alta prevalência de postura inadequada é o ato de pegar objetos do chão, realizado tanto no ambiente escolar, quanto no doméstico e esportivo (NOLL et al., 2013a), podendo provocar sérios danos à estrutura do disco intervertebral quando realizado de maneira incorreta (WILKE et al., 1999; STRAKER, 2003). Muitos estudantes não realizam essa atividade de maneira correta, ou seja, a partir da técnica de agachamento, com flexão de joelhos e quadril, optando por realizá-la com os joelhos estendidos e com flexão da coluna (STRAKER, 2003). Nesse sentido, especula-se que o motivo da não utilização da técnica adequada para levantar um objeto do chão esteja relacionado à tendência natural do ser humano em realizar as suas atividades em um menor gasto energético possível, que não é o caso do levantamento com agachamento, onde a fadiga pode ser induzida mais precocemente (NOLL et al., 2012).

Em contrapartida, alguns hábitos apresentaram alta prevalência de posturas adequadas, como o ato de dormir e o modo de transportar o material escolar. O cuidado com o transporte da mochila escolar tem sido um dos principais objetos de atenção da comunidade científica que investiga a saúde escolar, principalmente no que concerne a alterações posturais e dores nas costas entre crianças e adolescentes em idade escolar. Por exemplo, já está bem documentado que, a grande maioria dos escolares, em diferentes localidades, utiliza a mochila com duas alças, sobre os dois ombros ao transportar o material escolar (KELLIS; EMMANOUILIDOU, 2010; NOLL et al., 2013a). No presente estudo, especula-se que esse resultado positivo pode ser efeito da prática docente dos professores do Colégio de Aplicação, que rotineiramente ressaltam em suas aulas a importância de hábitos adequados nas AVD's, principalmente no modo de transportar a mochila, ou ainda pela grande ênfase dada pela mídia a este assunto (NOLL et al., 2013a). Embora, especificamente nesse colégio não estejam sendo desenvolvidas ações preventivas para os hábitos posturais inadequados, tem sido reportado efeitos positivos de programas preventivos realizados em outras localidades, nos últimos anos, para o ensinamento dos hábitos posturais (FERNANDES; CASAROTTO; JOÃO, 2008; CANDOTTI; LEMOS; NOLL, 2011; NOLL; CANDOTTI; VIEIRA, 2012a). Assim, entende-se que os resultados do presente estudo podem balizar o desenvolvimento de futuras ações preventivas no Colégio de Aplicação, uma vez que fornece um retrato da realidade local.

Em relação à postura para dormir, as posições de decúbito dorsal e ventral foram consideradas inadequadas e, somente, a posição de decúbito lateral foi considerada adequada. A grande maioria dos escolares indicou dormir na posição adequada, divergindo de estudo anterior, no qual foi encontrado que $66,4 \%$ dos escolares dormiam na posição incorreta (REBOLHO et al., 2011). Entretanto, corroborando com os nossos achados, também foram encontradas altas prevalências (acima dos 65\%) de escolares que dormiam na posição correta, em uma cidade do interior do estado do Rio Grande do Sul (NOLL et al., 2013a; NOLL et al., 2013b).

Diferente da nossa expectativa, a postura adequada para dormir apresentou associação com dor nas costas. A pergunta do questionário BackPEI que se refere à postura de dormir, questiona ao indivíduo apenas qual o decúbito do corpo durante o sono, porém não investiga a posição de cada segmento corporal. Assim, especula-se que, mesmo estando na posição de decúbito lateral, o escolar poderia estar mantendo algum dos segmentos corporais desalinhados, ou seja, em postura inadequada. Para uma adequada postura de dormir, o indivíduo deve estar com as pernas paralelas, de preferência com um travesseiro entre elas, permitindo a posição correta dos membros inferiores e da coluna lombar; os braços também devem estar apoiados em um travesseiro, permitindo que a coluna vertebral não tenha nenhuma rotação. Além disso, a posição da coluna cervical deve estar alinhada com o restante da coluna vertebral, sem nenhum tipo de inclinação 
(KENDALL et al., 2007). Neste sentido, caso algum dos segmentos corporais esteja posicionado de maneira inadequada, pode causar dor e desconforto, além do desenvolvimento de alterações posturais (KENDALL et al., 2007).

Em suma, os resultados do presente estudo vêm confirmar os achados da literatura, demostrando a necessidade de serem adotados programas educativos de promoção da saúde dos escolares, com ações voltadas para a educação postural. Portanto, partindo do pressuposto de que ao realizar a identificação precoce dos hábitos posturais inadequados e da dor nas costas, torna-se possível a realização dessas ações de caráter preventivo, tais como as Escolas Posturais (NOLL et al., 2012a), influenciando positivamente os hábitos posturais dos escolares (CANDOTTI et al., 2010). Além disso, entende-se que o desenvolvimento de mais estudos dessa natureza, em diferentes regiões do estado e com caráter longitudinal, possibilitará a compreensão dos fatores associados à dor e poderá ser utilizado para subsidiar políticas públicas estaduais de saúde escolar.

\section{Considerações finais}

A prevalência de dor nas costas nos escolares do Ensino Fundamental do Colégio de Aplicação da UFRGS é de 55,3\%, sendo que as meninas têm significativamente maior chance de apresentar dor em relação aos meninos. Quanto aos hábitos posturais, existe prevalência superior a $90 \%$ de posturas inadequadas nas AVD's pegar objeto do chão e sentar (para escrever, para conversar com amigos e para utilizar o computador). Em contrapartida, os resultados demonstraram prevalência superior a $50 \%$ de hábitos posturais adequados nas AVD's transportar o material escolar e dormir. A dor nas costas está associada ao hábito de sentar para escrever inadequadamente e de dormir adequadamente, resultado explicado no presente estudo. Considerando a realidade descrita, entende-se que seria importante a realização de ações de caráter preventivo nesse ambiente escolar para impactar positivamente sobre os hábitos posturais dos escolares.

\section{Referências}

AUVINEN, Juha et al. Is insufficient quantity and quality of sleep a risk factor for neck, shoulder and low back pain? A longitudinal study among adolescents. European Spine Journal, São Paulo, v. 19, p. 641-649, abr. 2010.

BUENO, Rita de Cássia; RECH, Ricardo Rodrigo. Desvios posturais em escolares de uma cidade do Sul do Brasil. Revista Paulista de Pediatria, São Paulo, v. 31, n. 2, jun. 2013.

BRACCIALLI, Lígia Maria Presumido; VILARTA, Roberto. Aspectos a serem considerados na elaboração de programas de prevenção e orientação de problemas posturais. Revista Paulista de Educação Física, v. 2, n. 14, p. 159-171, jul./dez. 2000.

CANDOTTI, Cláudia Tarragô et al. Escola de postura: uma metodologia adaptada aos pubescentes. Revista Mackenzie de Educação Física e Esporte, v. 9, n. 2, p. 91-100, 2010.

CANDOTTI, Cláudia Tarragô; NOLL, Matias; CRUZ, Melissa. Prevalência de dor lombar e os desequilíbrios musculares em manicures. Revista Arquivos em Movimento, Rio de Janeiro, v. 6, n.1, p.125-140, 2010.

CANDOTTI, Cláudia Tarragô; LEMOS Andréa Plocharski Ambrosi de; NOLL, Matias. Escola postural para crianças de 10 a 14 anos inserida no contexto do ensino fundamental. Revista Brasileira de Ciência e Movimento, v. 19, n. 2, p. 33-44, 2011.

CARVALHO, Valdemir Galvão; SANTOS, Varélio Gomes; CARVALHO, Verônica Galvão. Associação entre sensação de dor e desconforto pelos segmentos corporais, postura sentada do aluno 
em sala de aula e o mobiliário escolar (cadeira/mesa). Revista Educação em Questão, Natal, v. 33, n. 19, p. 35-62, set./dez. 2008.

DETSCH, Cíntia et al. Prevalência de alterações posturais em escolares do ensino médio em uma cidade no Sul do Brasil. Revista Panamericana de Salud Publica, Washington, v. 21, n. 4, p. 231238, abr. 2007.

FERNANDES, Susi Mary de Souza; CASAROTTO, Raquel Aparecida; JOÃO, Silvia Maria Amado. Effects of educational sessions on school backpack use among elementary school students. Revista Brasileira de Fisioterapia, São Carlos, v. 6, p. 447-453, nov./dez. 2008.

FERREIRA, Gustavo et al. Prevalence and associated factors of back pain in adults from Southern Brazil: a population-based study. Revista Brasileira de Fisioterapia, v. 15, n.1, p. 31-36, jan./fev. 2011.

FREIRE, Ivete de Aquino; TEIXEIRA, Tatiane Gomes; SALES, Célio Reis. Hábitos posturais: diagnóstico a partir de fotografias. Conexões, Campinas, v. 6, n. 2, p. 28-41, 2008.

HOY, Damian et al. The epidemiology of low back pain. Best Practice \& Research Clinical Rheumatology, v. 24, p. 769-81, 2010.

KELLIS, Eleftherios; EMMANOUILIDOU, Maria. The effects of age and gender on the weight and use of schoolbags. Pediatric Physical Therapy, v. 22, p. 17-45, 2010.

KENDALL, Florence Peterson et al. Músculos, provas e funções. 5. ed. São Paulo: Manole; 2007.

KOROVESSIS, Panagiotis et al. Backpacks, back pain, sagittal spinal curves and trunk alignment in adolescents. Spine, v. 30, n. 2, p. 247-55, jan. 2005.

KUNZLER, Mateus. Postura sentada e alterações posturais da coluna vertebral no plano sagital de escolares da cidade de Lajeado/RS, Brasil. 2013. 38f. Trabalho de Conclusão de Curso (Licenciatura em Educação Física) - Universidade Federal do Rio Grande do Sul, Porto Alegre, 2013.

LIS, Angela Maria; BLACK, Katia; NORDIN, Margareta. Association between sitting and occupational LBP. European Spine Journal, v. 16, p. 283-289, fev. 2007.

MARTÍNEZ-CRESPO, Garcia et al. Dolor de espalda em adolescentes: prevalência y fatores associados. Rehabilitacion, v. 43, n. 2, p. 72-80, 2009.

NAJENSON, Deborah Alperovitch et al. Low back pain among professional bus drivers: ergonomic and occupational-psychosocial risk factors. Israel Medical Association Journal, v. 12, p. 26-31, jan. 2010.

NOLL, Matias et al. Back Pain and Body Posture Evaluation Instrument (BackPEI): Development, Content Validation and Reproducibility. International Journal of Public Health, Basel, v. 58, p. 565-72, 2013.

NOLL, Matias; CANDOTTI, Cláudia Tarragô; TIGGEMANN, Carlos Leandro; SCHOENELL, Maira Cristina Wolf; VIEIRA, Adriane. Prevalência de hábitos posturais inadequados de escolares do ensino fundamental da cidade de Teutônia: um estudo de base populacional. Revista Brasileira de Ciências do Esporte, Florianópolis, v. 35, n. 4, p. 983-1004, out./dez. 2013a.

NOLL, Matias et al. Dor nas costas, hábitos posturais e comportamentais de escolares da rede municipal de ensino de Teutônia/RS. Journal of Human Growth and Development, v. 23, n. 2, p. $1-10,2013 b$.

NOLL, Matias et al. Prevalência de dor nas costas e fatores associados em escolares do Ensino Fundamental do município de Teutônia/RS. Revista Brasileira de Saúde Materno Infantil, Recife, v. 12, n. 4 , p. 395-402 out./dez. 2012.

NOLL, Matias; CANDOTTI, Cláudia Tarragô; VIEIRA, Adriane. Escola postural: revisão sistemática dos programas desenvolvidos para escolares do Brasil. Movimento. v. 18, n. 4, p. 265-291, 2012a.

REBOLHO, Marilia Christina Tenório et al. Prevalence of musculoskeletal pain and perception of postural habits among primary school students. Revista Médica, São Paulo, v. 90, n. 2, p. 68-77, abr./jun. 2011.

SILVA, Priscila Rejane Lima. Prevalência de hábitos posturais adequados e de dor nas costas em escolares de Ensino Médio da Rede Estadual do município de Esteio/RS. 2013. 30 f. Trabalho de Conclusão de Curso (Licenciatura em Educação Física) - Universidade Federal do Rio Grande do Sul, Porto Alegre, 2013. 
SHEHAB, Dia; JARALLAH, Khaled. Nonspecific low-back pain in Kuwaiti children and adolescents: associated factors. Journal of Adolescents Health, v. 36, p. 32-35, 2005.

SKOFFER, Birgit. Low Back Pain in 15 to 16 year old children in relation to school furniture and carrying of the school bag. Spine, v. 32, p. 713-717, nov. 2007.

STRAKER, Leon. Evidence to support using squat, semi-squat and stoop techniques to lift low-lying objects. International Journal of Industrial Ergonomics, New York, v. 31, n. 3, p. 149-160, mar. 2003.

VITTA, Alberto de et al. Prevalência e fatores associados à dor lombar em escolares. Caderno de Saúde Pública, v. 27, p. 1520-1528, ago. 2011.

ZAPATA, Aura Ligia et al. Pain and musculoskeletal pain syndromes related to computer and video game use in adolescents. European Journal of Pediatrics, v. 165, p. 408-414, 2006.

WATSON, Kath et al. Low back pain in schoolchildren: occurrence and characteristics. Pain, v. 97, p. 87-92, 2002.

WILKE, Hans Joachim et al. New in vivo measurements of pressures in the intervertebral disc in daily life. Spine, v. 24, n. 8, p. 755-762, 1999. 